\title{
IMPLICIT PREDICTOR-CORRECTOR ITERATION PROCESS FOR FINITELY MANY ASYMPTOTICALLY (QUASI-)NONEXPANSIVE MAPPINGS
}

\author{
L. C. CENG, N. C. WONG, AND J. C. YAO
}

Received 13 February 2006; Revised 3 June 2006; Accepted 5 June 2006

We study an implicit predictor-corrector iteration process for finitely many asymptotically quasi-nonexpansive self-mappings on a nonempty closed convex subset of a Banach space $E$. We derive a necessary and sufficient condition for the strong convergence of this iteration process to a common fixed point of these mappings. In the case $E$ is a uniformly convex Banach space and the mappings are asymptotically nonexpansive, we verify the weak (resp., strong) convergence of this iteration process to a common fixed point of these mappings if Opial's condition is satisfied (resp., one of these mappings is semicompact). Our results improve and extend earlier and recent ones in the literature.

Copyright (c) 2006 L. C. Ceng et al. This is an open access article distributed under the Creative Commons Attribution License, which permits unrestricted use, distribution, and reproduction in any medium, provided the original work is properly cited.

\section{Introduction and preliminaries}

Let $E$ be a real Banach space equipped with norm $\|\cdot\|$, let $C$ be a nonempty subset of $E$, and let $T: C \rightarrow C$. The set $F(T)=\{x \in C: T x=x\}$ consists of all fixed points of $T$.

Definition 1.1. T is said to be

(1) nonexpansive if

$$
\|T x-T y\| \leq\|x-y\|, \quad \forall x, y \in C
$$

(2) asymptotically nonexpansive [3] if there exists a sequence $\left\{k_{n}\right\}_{n=1}^{\infty} \subset[1, \infty)$ with $\lim _{n \rightarrow \infty} k_{n}=1$ such that

$$
\left\|T^{n} x-T^{n} y\right\| \leq k_{n}\|x-y\|, \quad \forall x, y \in C, n \geq 1 ;
$$

(3) asymptotically quasi-nonexpansive if $F(T) \neq \varnothing$, and there exists a sequence $\left\{k_{n}\right\}_{n=1}^{\infty} \subset$ $[1, \infty)$ with $\lim _{n \rightarrow \infty} k_{n}=1$ such that

$$
\left\|T^{n} x-p\right\| \leq k_{n}\|x-p\|, \quad \forall x \in C, p \in F(T), n \geq 1 ;
$$

Hindawi Publishing Corporation Journal of Inequalities and Applications Volume 2006, Article ID 65983, Pages 1-11 
(4) semicompact [9] if for any bounded sequence $\left\{x_{n}\right\} \subset C$ with $\lim _{n \rightarrow \infty}\left\|x_{n}-T x_{n}\right\|=$ 0 , there exists a strongly convergent subsequence of $\left\{x_{n}\right\}$.

The class of asymptotically nonexpansive mappings, as a natural extension of that of nonexpansive mappings, was introduced by Goebel and Kirk [3]. They proved that if $C$ is a nonempty bounded closed convex subset of a uniformly convex Banach space $E$, then every asymptotically nonexpansive self-mapping $T$ on $C$ has a fixed point. Furthermore, the study of iterative construction for fixed points of asymptotically nonexpansive mappings began in 1978. Bose [1] first proved that if the uniformly convex Banach space $E$ satisfies Opial's condition [5], then $\left\{T^{n} x\right\}$ converges weakly to a fixed point of $T$, provided $T$ is asymptotically regular at $x$, that is, $\lim _{n \rightarrow \infty}\left\|T^{n} x-T^{n+1} x\right\|=0$. A Banach space $E$ is said to satisfy Opial's condition [5] if whenever $\left\{x_{n}\right\}$ is a sequence in $E$ which converges weakly to $x$, one has

$$
\liminf _{n \rightarrow \infty}\left\|x_{n}-x\right\|<\liminf _{n \rightarrow \infty}\left\|x_{n}-y\right\|, \quad \forall y \in E, y \neq x .
$$

It is well known that every Hilbert space satisfies Opial's condition (see, e.g., [5]).

$\mathrm{Xu}$ and Ori [8] first introduced an implicit iteration process for $N$ nonexpansive mappings in a Hilbert space and proved the following weak convergence theorem.

Theorem 1.2 (see [8]). Let $H$ be a Hilbert space and let $C$ be a nonempty closed convex subset of $H$. Let $\left\{T_{i}\right\}_{i=1}^{N}$ be $N$ nonexpansive self-mappings on $C$ such that $F=\bigcap_{i=1}^{N} F\left(T_{i}\right) \neq$ $\varnothing$. Let $x_{0} \in C$ and let $\left\{\alpha_{n}\right\}_{n=1}^{\infty}$ be a sequence in $(0,1)$ such that $\lim _{n \rightarrow \infty} \alpha_{n}=0$. Then the sequence $\left\{x_{n}\right\}$ defined implicity by

$$
x_{n}=\alpha_{n} x_{n-1}+\left(1-\alpha_{n}\right) T_{n(\bmod N)} x_{n}, \quad n \geq 1,
$$

converges weakly to a common fixed point of mappings $\left\{T_{j}\right\}_{j=1}^{N}$.

Later, Sun [7] introduced and studied another implicit iteration process

$$
x_{n}=\alpha_{n} x_{n-1}+\left(1-\alpha_{n}\right) T_{n(\bmod N)}^{l_{n}+1} x_{n}, \quad n \geq 1
$$

for $N$ asymptotically quasi-nonexpansive self-mappings $\left\{T_{j}\right\}_{j=1}^{N}$ on a nonempty bounded closed convex subset $C$ of a Banach space $E$, where $\left\{\alpha_{n}\right\}$ is a sequence in $(0,1), x_{0}$ is an initial point in $C$, and $n=l_{n} N+n(\bmod N)$. Moreover, he proved that the sequence $\left\{x_{n}\right\}$ defined by his iteration process converges strongly to a common fixed point of $\left\{T_{j}\right\}_{j=1}^{N}$ under suitable conditions.

At the same time, in [10], Zhou and Chang introduced and studied the following implicit iteration process:

$$
x_{n}=\alpha_{n} x_{n-1}+\beta_{n} T_{n(\bmod N)}^{n} x_{n}+\gamma_{n} u_{n}, \quad n \geq 1,
$$

for $N$ asymptotically nonexpansive self-mappings $\left\{T_{j}\right\}_{j=1}^{N}$ on a nonempty closed convex subset $C$ of a Banach space $E$, where $\left\{\alpha_{n}\right\},\left\{\beta_{n}\right\},\left\{\gamma_{n}\right\}$ are three sequences in $[0,1], x_{0}$ is an initial point in $C$, and $\left\{u_{n}\right\}$ is a bounded sequence in $C$. Moreover, they proved that the sequence $\left\{x_{n}\right\}$ defined by their iteration process converges weakly to a common fixed point of $\left\{T_{j}\right\}_{j=1}^{N}$ under suitable conditions. 
As indicated in [10], if $T_{1}, T_{2}, \ldots, T_{N}: C \rightarrow C$ are $N$ asymptotically nonexpansive mappings, then there exists a sequence, called common Lipschitz constants, $\left\{k_{n}\right\} \subset[1, \infty)$ with $\lim _{n \rightarrow \infty} k_{n}=1$ such that for each $i=1,2, \ldots, N$,

$$
\left\|T_{i}^{n} x-T_{i}^{n} y\right\| \leq k_{n}\|x-y\|, \quad \forall x, y \in C, n \geq 1 .
$$

A similar situation occurs when $T_{1}, T_{2}, \ldots, T_{N}$ are asymptotically quasi-nonexpansive. By convention, we write $T_{n}:=T_{n(\bmod N)}$, for integer $n \geq 1$, with the mod function taking values in the set $\{1,2, \ldots, N\}$. In other words, if $n=l_{n} N+q$ for some unique integers $l_{n} \geq 0$ and $1 \leq q \leq N$, then we set $T_{n}=T_{q}$.

In this paper, we introduce the following implicit predictor-corrector iteration process with an auxiliary finite family of asymptotically quasi-nonexpansive self-mappings on $C$.

Definition 1.3 (basic setup). Let $C$ be a nonempty closed convex subset of a Banach space $E$, and let $\left\{T_{1}, T_{2}, \ldots, T_{N}\right\}$ and $\left\{\widehat{T}_{1}, \widehat{T}_{2}, \ldots, \widehat{T}_{\hat{N}}\right\}$ be two families of asymptotically quasinonexpansive mappings from $C$ into $C$ with common Lipschitz constants $\left\{k_{n}\right\}$ and $\left\{\hat{k}_{n}\right\}$ such that $\sum_{n=1}^{\infty}\left(k_{n}-1\right)<+\infty$ and $\sum_{n=1}^{\infty}\left(\hat{k}_{n}-1\right)<+\infty$, respectively. Let $\left\{x_{n}\right\}$ be an iterative sequence in $C$ generated from an arbitrary $x_{0} \in C$ by the following three steps.

Auxiliary step. With $x_{n-1}(n \geq 1)$ established, $y_{n}$ is computed implicitly by

$$
y_{n}=\hat{\alpha}_{n} x_{n-1}+\hat{\beta}_{n} \widehat{T}_{n}^{\hat{l}_{n}} y_{n}+\hat{\gamma}_{n} \hat{u}_{n}
$$

Predictor step. With $y_{n}$ obtained in the auxiliary step, $z_{n}$ is computed implicitly by

$$
z_{n}=\bar{\alpha}_{n} y_{n}+\bar{\beta}_{n} T_{n}^{l_{n}} z_{n}+\bar{\gamma}_{n} \bar{u}_{n}
$$

Corrector step. With $z_{n}$ obtained in the predictor step, $x_{n}$ is computed explicitly by

$$
x_{n}=\alpha_{n} y_{n}+\beta_{n} T_{n}^{l_{n}} z_{n}+\gamma_{n} u_{n} .
$$

Here, $T_{n}:=T_{n(\bmod N)}$ and $\widehat{T}_{n}:=\widehat{T}_{n(\bmod \hat{N})}$ for $n=1,2, \ldots$ On the other hand, $\left\{u_{n}\right\}_{n=1}^{\infty}$, $\left\{\hat{u}_{n}\right\}_{n=1}^{\infty},\left\{\bar{u}_{n}\right\}_{n=1}^{\infty}$ are three bounded sequences in $C$; and $\left\{\alpha_{n}\right\}_{n=1}^{\infty},\left\{\hat{\alpha}_{n}\right\}_{n=1}^{\infty},\left\{\bar{\alpha}_{n}\right\}_{n=1}^{\infty}$, $\left\{\beta_{n}\right\}_{n=1}^{\infty},\left\{\hat{\beta}_{n}\right\}_{n=1}^{\infty},\left\{\bar{\beta}_{n}\right\}_{n=1}^{\infty},\left\{\gamma_{n}\right\}_{n=1}^{\infty},\left\{\hat{\gamma}_{n}\right\}_{n=1}^{\infty},\left\{\bar{\gamma}_{n}\right\}_{n=1}^{\infty}$ are nine real sequences in $[0,1]$ such that

$$
\begin{gathered}
\alpha_{n}+\beta_{n}+\gamma_{n}=1 \quad(\forall n \geq 1), \quad \sum_{n=1}^{\infty} \gamma_{n}<+\infty, \\
\hat{\alpha}_{n}+\hat{\beta}_{n}+\hat{\gamma}_{n}=1 \quad(\forall n \geq 1), \quad \sum_{n=1}^{\infty} \hat{\gamma}_{n}<+\infty, \\
\bar{\alpha}_{n}+\bar{\beta}_{n}+\bar{\gamma}_{n}=1 \quad(\forall n \geq 1), \quad \sum_{n=1}^{\infty} \bar{\gamma}_{n}<+\infty, \\
0<\hat{\beta}_{n}, \bar{\beta}_{n} \leq c<K^{-1} \quad(\forall n \geq 1), \quad K=\max \left\{\sup _{n \geq 1} k_{n}, \sup _{n \geq 1} \hat{k}_{n}\right\} \geq 1 .
\end{gathered}
$$


Remark 1.4. Since $0<\hat{\beta}_{n}, \bar{\beta}_{n} \leq c<K^{-1}$, it is clear that the mappings $y \mapsto \hat{\alpha}_{n} x_{n-1}+\hat{\beta}_{n} \widehat{T}_{n} \hat{l}_{n} y+$ $\hat{\gamma}_{n} \hat{u}_{n}$ and $z \mapsto \bar{\alpha}_{n} y_{n}+\bar{\beta}_{n} T_{n}^{l_{n}} z+\bar{\gamma}_{n} \bar{u}_{n}$ are two contractions from the nonempty closed convex set $C$ into itself. Thus, by the Banach contraction principle, there exist the unique points $y_{n}, z_{n} \in C$ such that (1.9a) and (1.9b) hold, respectively. Therefore, the sequence $\left\{x_{n}\right\}$ is well defined.

Our aim is to consider and study the strong and weak convergences of the above implicit predictor-corrector iteration process. To this end, we need the following lemmas.

Lemma 1.5. Let $\left\{b_{n}\right\},\left\{\bar{b}_{n}\right\},\left\{\hat{b}_{n}\right\}$ be three nonnegative real sequences with finite sums. Then $\sum_{n=1}^{\infty} \lambda_{n}<+\infty$, where $\lambda_{n}=\left(1+b_{n}\right)\left(1+\bar{b}_{n}\right)\left(1+\hat{b}_{n}\right)-1$ for each $\geq 1$.

Lemma 1.6 (see [10]). Let $\left\{a_{n}\right\},\left\{\lambda_{n}\right\},\left\{\mu_{n}\right\}$ be three nonnegative real sequences such that $\sum_{n=1}^{\infty} \lambda_{n}<+\infty, \sum_{n=1}^{\infty} \mu_{n}<+\infty$, and

$$
a_{n+1} \leq\left(1+\lambda_{n}\right) a_{n}+\mu_{n}, \quad \forall n \geq 1 .
$$

Then $\lim _{n \rightarrow \infty} a_{n}$ exists.

Lemma 1.7 (see [6]). Let $E$ be a uniformly convex Banach space, $\left\{t_{n}\right\} \subset[b, c] \subset(0,1)$, and $\left\{x_{n}\right\},\left\{y_{n}\right\} \subset E$. If $\lim _{n \rightarrow \infty}\left\|t_{n} x_{n}+\left(1-t_{n}\right) y_{n}\right\|=d<+\infty$, $\limsup _{n \rightarrow \infty}\left\|x_{n}\right\| \leq d$, and $\limsup _{n \rightarrow \infty}\left\|y_{n}\right\| \leq d$, then $\lim _{n \rightarrow \infty}\left\|x_{n}-y_{n}\right\|=0$.

Lemma 1.8 (demiclosed principle [2]). Let E be a uniformly convex Banach space, let $C$ be a nonempty closed convex subset of $E$, and let $T: C \rightarrow C$ be an asymptotically nonexpansive mapping with $F(T) \neq \varnothing$. Then $I-T$ is demiclosed at zero, that is, for any sequence $\left\{x_{n}\right\} \subset$ C,

$$
\begin{gathered}
x_{n} \longrightarrow q \in C \text { weakly } \\
(I-T) x_{n} \longrightarrow 0 \text { strongly }
\end{gathered} \Longrightarrow(I-T) q=0 .
$$

\section{Main results}

LEMMA 2.1. Let $C$ be a nonempty closed convex subset of a Banach space $E$, and let $\left\{T_{i}\right\}_{i=1}^{N}$ and $\left\{\widehat{T}_{j}\right\}_{j=1}$ be two finite families of asymptotically quasi-nonexpansive self-mappings on $C$ such that $\bigcap_{i=1}^{N} F\left(T_{i}\right) \cap \bigcap_{j=1}^{\hat{N}} F\left(\widehat{T}_{j}\right) \neq \varnothing$. If $\left\{x_{n}\right\},\left\{y_{n}\right\}$, and $\left\{z_{n}\right\}$ are the iterative sequences defined by (1.9a), (1.9b), and (1.9c), then for each $p \in \bigcap_{i=1}^{N} F\left(T_{i}\right) \cap \bigcap_{j=1}^{\hat{N}} F\left(\widehat{T}_{j}\right)$, there hold

$$
\lim _{n \rightarrow \infty}\left\|x_{n}-p\right\|=d, \quad \quad \limsup _{n \rightarrow \infty}\left\|y_{n}-p\right\| \leq d, \quad \limsup _{n \rightarrow \infty}\left\|z_{n}-p\right\| \leq d .
$$

Proof. Since $\left\{u_{n}\right\}_{n=1}^{\infty},\left\{\hat{u}_{n}\right\}_{n=1}^{\infty},\left\{\bar{u}_{n}\right\}_{n=1}^{\infty}$ are three bounded sequences in $C$, for any given $p \in \bigcap_{i=1}^{N} F\left(T_{i}\right) \cap \bigcap_{j=1}^{\hat{N}} F\left(\widehat{T}_{j}\right)$, we have

$$
M:=\max \left\{\sup _{n \geq 1}\left\|u_{n}-p\right\|, \sup _{n \geq 1}\left\|\hat{u}_{n}-p\right\|, \sup _{n \geq 1}\left\|\bar{u}_{n}-p\right\|\right\}<+\infty .
$$


Note that $1-\bar{\beta}_{n} k_{l_{n}} \geq 1-c K>0$ and $1-\hat{\beta}_{n} \hat{k}_{\hat{l}_{n}} \geq 1-c K>0$. Put

$$
L=\frac{1}{1-c K}, \quad b_{n}=\beta_{n}\left(k_{l_{n}}-1\right), \quad \bar{b}_{n}=\frac{1-\bar{\beta}_{n}}{1-\bar{\beta}_{n} k_{l_{n}}}-1, \quad \hat{b}_{n}=\frac{1-\hat{\beta}_{n}}{1-\hat{\beta}_{n} \hat{k}_{\hat{l}_{n}}}-1 .
$$

Then we have

$$
\begin{aligned}
& 0 \leq b_{n}=\beta_{n}\left(k_{l_{n}}-1\right) \leq k_{l_{n}}-1, \quad 1+b_{n} \leq K, \\
& 0 \leq \bar{b}_{n}=\frac{\bar{\beta}_{n}\left(k_{l_{n}}-1\right)}{1-\bar{\beta}_{n} k_{l_{n}}} \leq L\left(k_{l_{n}}-1\right), \quad 1+\bar{b}_{n} \leq L, \\
& 0 \leq \hat{b}_{n}=\frac{\hat{\beta}_{n}\left(\hat{k}_{\hat{l}_{n}}-1\right)}{1-\hat{\beta}_{n} \hat{k}_{\hat{l}_{n}}} \leq L\left(\hat{k}_{\hat{l}_{n}}-1\right), \quad 1+\hat{b}_{n} \leq L .
\end{aligned}
$$

Observe that

$$
\begin{aligned}
\left\|y_{n}-p\right\| & =\left\|\hat{\alpha}_{n}\left(x_{n-1}-p\right)+\hat{\beta}_{n}\left(\hat{T}_{n}^{\hat{l}_{n}} y_{n}-p\right)+\hat{\gamma}_{n}\left(\hat{u}_{n}-p\right)\right\| \\
& \leq \hat{\alpha}_{n}\left\|x_{n-1}-p\right\|+\hat{\beta}_{n} \hat{k}_{\hat{l}_{n}}\left\|y_{n}-p\right\|+\hat{\gamma}_{n}\left\|\hat{u}_{n}-p\right\| .
\end{aligned}
$$

It follows

$$
\begin{aligned}
\left\|y_{n}-p\right\| & \leq \frac{\hat{\alpha}_{n}}{1-\hat{\beta}_{n} \hat{k}_{\hat{l}_{n}}}\left\|x_{n-1}-p\right\|+\frac{\hat{\gamma}_{n}}{1-\hat{\beta}_{n} \hat{k}_{\hat{l}_{n}}}\left\|\hat{u}_{n}-p\right\| \\
& \leq \frac{1-\hat{\beta}_{n}}{1-\hat{\beta}_{n} \hat{k}_{\hat{l}_{n}}}\left\|x_{n-1}-p\right\|+L M \hat{\gamma}_{n} \\
& =\left(1+\hat{b}_{n}\right)\left\|x_{n-1}-p\right\|+L M \hat{\gamma}_{n} .
\end{aligned}
$$

Similarly,

$$
\begin{aligned}
\left\|z_{n}-p\right\| & =\left\|\bar{\alpha}_{n}\left(y_{n}-p\right)+\bar{\beta}_{n}\left(T_{n}^{l_{n}} z_{n}-p\right)+\bar{\gamma}_{n}\left(\bar{u}_{n}-p\right)\right\| \\
& \leq \bar{\alpha}_{n}\left\|y_{n}-p\right\|+\bar{\beta}_{n} k_{l_{n}}\left\|z_{n}-p\right\|+\bar{\gamma}_{n}\left\|\bar{u}_{n}-p\right\|
\end{aligned}
$$

Consequently,

$$
\begin{aligned}
\left\|z_{n}-p\right\| & \leq \frac{\bar{\alpha}_{n}}{1-\bar{\beta}_{n} k_{l_{n}}}\left\|y_{n}-p\right\|+\frac{\bar{\gamma}_{n}}{1-\bar{\beta}_{n} k_{l_{n}}}\left\|\bar{u}_{n}-p\right\| \\
& \leq \frac{1-\bar{\beta}_{n}}{1-\bar{\beta}_{n} k_{l_{n}}}\left\|y_{n}-p\right\|+L M \bar{\gamma}_{n} \\
& =\left(1+\bar{b}_{n}\right)\left\|y_{n}-p\right\|+L M \bar{\gamma}_{n} .
\end{aligned}
$$


Therefore,

$$
\begin{aligned}
\left\|x_{n}-p\right\| & =\left\|\alpha_{n}\left(y_{n}-p\right)+\beta_{n}\left(T_{n}^{l_{n}} z_{n}-p\right)+\gamma_{n}\left(u_{n}-p\right)\right\| \\
& \leq \alpha_{n}\left\|y_{n}-p\right\|+\beta_{n} k_{l_{n}}\left\|z_{n}-p\right\|+\gamma_{n}\left\|u_{n}-p\right\| \\
& \leq\left(1-\beta_{n}\right)\left\|y_{n}-p\right\|+\beta_{n} k_{l_{n}}\left[\left(1+\bar{b}_{n}\right)\left\|y_{n}-p\right\|+L M \bar{\gamma}_{n}\right]+\gamma_{n} M \\
& \leq\left(1+\beta_{n}\left(k_{l_{n}}-1\right)\right)\left(1+\bar{b}_{n}\right)\left\|y_{n}-p\right\|+M\left[K L \bar{\gamma}_{n}+\gamma_{n}\right] \\
& \leq\left(1+b_{n}\right)\left(1+\bar{b}_{n}\right)\left\|y_{n}-p\right\|+K L M\left[\bar{\gamma}_{n}+\gamma_{n}\right] \\
& \leq\left(1+b_{n}\right)\left(1+\bar{b}_{n}\right)\left[\left(1+\hat{b}_{n}\right)\left\|x_{n-1}-p\right\|+L M \hat{\gamma}_{n}\right]+K L M\left[\bar{\gamma}_{n}+\gamma_{n}\right] \\
& \leq\left(1+b_{n}\right)\left(1+\bar{b}_{n}\right)\left(1+\hat{b}_{n}\right)\left\|x_{n-1}-p\right\|+K L^{2} M \hat{\gamma}_{n}+K L M\left[\bar{\gamma}_{n}+\gamma_{n}\right] \\
& \leq\left(1+b_{n}\right)\left(1+\bar{b}_{n}\right)\left(1+\hat{b}_{n}\right)\left\|x_{n-1}-p\right\|+K L^{2} M\left[\gamma_{n}+\bar{\gamma}_{n}+\hat{\gamma}_{n}\right] \\
& =\left(1+\lambda_{n}\right)\left\|x_{n-1}-p\right\|+\mu_{n},
\end{aligned}
$$

where $\lambda_{n}=\left(1+b_{n}\right)\left(1+\bar{b}_{n}\right)\left(1+\hat{b}_{n}\right)-1$, and $\mu_{n}=K L^{2} M\left[\gamma_{n}+\bar{\gamma}_{n}+\hat{\gamma}_{n}\right]$.

Since $\sum_{n=1}^{\infty}\left(k_{l_{n}}-1\right)<+\infty$ and $\sum_{n=1}^{\infty}\left(\hat{k}_{\hat{l}_{n}}-1\right)<+\infty$, it follows from $(2.4)$ that $\sum_{n=1}^{\infty} b_{n}<$ $+\infty, \sum_{n=1}^{\infty} \bar{b}_{n}<+\infty$, and $\sum_{n=1}^{\infty} \hat{b}_{n}<+\infty$. Hence, we derive $\sum_{n=1}^{\infty} \lambda_{n}<+\infty$ by Lemma 1.5. Note that $\sum_{n=1}^{\infty} \gamma_{n}<+\infty, \sum_{n=1}^{\infty} \bar{\gamma}_{n}<+\infty$, and $\sum_{n=1}^{\infty} \hat{\gamma}_{n}<+\infty$. This provides $\sum_{n=1}^{\infty} \mu_{n}<+\infty$. By Lemma 1.6, $\lim _{n \rightarrow \infty}\left\|x_{n}-p\right\|$ exists. Let $\lim _{n \rightarrow \infty}\left\|x_{n}-p\right\|=d$.

Since $\lim _{n \rightarrow \infty} \hat{b}_{n}=\lim _{n \rightarrow \infty} \hat{\gamma}_{n}=0$, from (2.6), we obtain

$$
\limsup _{n \rightarrow \infty}\left\|y_{n}-p\right\| \leq \limsup _{n \rightarrow \infty}\left(1+\hat{b}_{n}\right)\left\|x_{n-1}-p\right\|+L M \limsup _{n \rightarrow \infty} \hat{\gamma}_{n} \leq d
$$

Further, since $\lim _{n \rightarrow \infty} \bar{b}_{n}=\lim _{n \rightarrow \infty} \bar{\gamma}_{n}=0$, from (2.8), we obtain

$$
\limsup _{n \rightarrow \infty}\left\|z_{n}-p\right\| \leq \limsup _{n \rightarrow \infty}\left(1+\bar{b}_{n}\right)\left\|y_{n}-p\right\|+L M \limsup _{n \rightarrow \infty} \bar{\gamma}_{n} \leq d
$$

Theorem 2.2. Let $C$ be a nonempty closed convex subset of a Banach space E. Let $\left\{T_{i}\right\}_{i=1}^{N}$ and $\left\{\widehat{T}_{j}\right\}_{j=1}^{\hat{N}}$ be two finite families of asymptotically quasi-nonexpansive self-mappings on $C$ such that $F:=\bigcap_{i=1}^{N} F\left(T_{i}\right) \cap \bigcap_{j=1}^{\hat{N}} F\left(\widehat{T}_{j}\right) \neq \varnothing$. Let $\left\{x_{n}\right\}$ be the iterative sequence defined by $(1.9 a),(1.9 b)$, and $(1.9 c)$. Then $\left\{x_{n}\right\}$ converges strongly to an element of $F$ if and only if

$$
\liminf _{n \rightarrow \infty} d\left(x_{n}, F\right)=0
$$

Proof. The necessity is obvious. For the sufficiency, we assume $\liminf _{n \rightarrow \infty} d\left(x_{n}, F\right)=0$. Let $p$ be any given element in $F$. Then from (2.9), we obtain

$$
\left\|x_{n}-p\right\| \leq\left(1+\lambda_{n}\right)\left\|x_{n-1}-p\right\|+\mu_{n}
$$


where $\sum_{n=1}^{\infty} \lambda_{n}<+\infty$ and $\sum_{n=1}^{\infty} \mu_{n}<+\infty$. Taking the infimum over all $p \in F$, we get

$$
d\left(x_{n}, F\right) \leq\left(1+\lambda_{n}\right) d\left(x_{n-1}, F\right)+\mu_{n} .
$$

Hence, $\lim _{n \rightarrow \infty} d\left(x_{n}, F\right)$ exists. Furthermore, we have $\lim _{n \rightarrow \infty} d\left(x_{n}, F\right)=0$.

By Lemma 2.1, we know that $\lim _{n \rightarrow \infty}\left\|x_{n}-p\right\|$ exists. Hence $\left\{x_{n}\right\}$ is bounded. Put $\delta_{n}=$ $\lambda_{n}\left\|x_{n-1}-p\right\|+\mu_{n}$. Then $\sum_{n=1}^{\infty} \delta_{n}<+\infty$, and (2.13) can be rewritten as

$$
\left\|x_{n}-p\right\| \leq\left\|x_{n-1}-p\right\|+\delta_{n}
$$

For arbitrary $\varepsilon>0$, choose $N_{0}$ such that $d\left(x_{N_{0}}, F\right)<\varepsilon / 4$ and $\sum_{j=N_{0}}^{\infty} \delta_{j}<\varepsilon / 4$. Consequently, for all $n, m \geq N_{0}$, we have

$$
\begin{aligned}
\left\|x_{n}-x_{m}\right\| & \leq\left\|x_{n}-p\right\|+\left\|x_{m}-p\right\| \\
& \leq\left\|x_{N_{0}}-p\right\|+\sum_{j=N_{0}+1}^{n} \delta_{j}+\left\|x_{N_{0}}-p\right\|+\sum_{j=N_{0}+1}^{m} \delta_{j} \\
& \leq 2\left\|x_{N_{0}}-p\right\|+2 \sum_{j=N_{0}}^{\infty} \delta_{j} .
\end{aligned}
$$

Taking the infimum over all $p \in F$, we obtain

$$
\left\|x_{n}-x_{m}\right\| \leq 2 d\left(x_{N_{0}}, F\right)+2 \sum_{j=N_{0}}^{\infty} \delta_{j} \leq \frac{2 \varepsilon}{4}+\frac{2 \varepsilon}{4}=\varepsilon .
$$

This shows that $\left\{x_{n}\right\}_{n=1}^{\infty}$ is Cauchy. Let $\lim _{n \rightarrow \infty} x_{n}=u$. It is easy to verify that $F$ is closed. Since $\lim _{n \rightarrow \infty} d\left(x_{n}, F\right)=0$, we must have that $u \in F$.

As a consequence of Lemma 2.1, the iterated sequence $\left\{x_{n}\right\}$ is bounded. If the underlying space $E$ is reflexive, then we can expect that its weak cluster points provide common fixed points of $T_{1}, T_{2}, \ldots, T_{N}$. This leads to the following theorem.

Theorem 2.3. Let E be a uniformly convex Banach space, let $C$ be a nonempty closed convex subset of E, and let $\left\{T_{i}\right\}_{i=1}^{N}$ (resp., $\left\{\widehat{T}_{j}\right\}_{j=1}^{\hat{N}}$ ) be a finite family of asymptotically nonexpansive (resp., asymptotically quasi-nonexpansive) self-mappings on $C$ such that $\bigcap_{j=1}^{\hat{N}} F\left(\widehat{T}_{j}\right) \cap$ $\bigcap_{i=1}^{N} F\left(T_{i}\right) \neq \varnothing$. Suppose $\lim _{n \rightarrow \infty} \hat{\beta}_{n}=0$ and $\left\{\beta_{n}\right\}_{n=1}^{\infty} \subset[b, c] \subset\left(0, K^{-1}\right)$, where $K$ is as in (1.10). Then every weak cluster point of the bounded iterative sequence $\left\{x_{n}\right\}$ defined by (1.9a), (1.9b), and (1.9c) belongs to $\bigcap_{i=1}^{N} F\left(T_{i}\right)$.

Proof. Let $p \in \bigcap_{j=1}^{\hat{N}} F\left(\widehat{T}_{j}\right) \cap \bigcap_{i=1}^{N} F\left(T_{i}\right)$. By Lemma 2.1, we have

$$
\lim _{n \rightarrow \infty}\left\|x_{n}-p\right\|=d, \quad \quad \limsup _{n \rightarrow \infty}\left\|y_{n}-p\right\| \leq d, \quad \quad \limsup _{n \rightarrow \infty}\left\|z_{n}-p\right\| \leq d
$$

Obviously, $\left\{x_{n}\right\},\left\{y_{n}\right\}$, and $\left\{z_{n}\right\}$ are bounded sequences in $C$. 
8 Implicit predictor-corrector iteration process

Observe that

$$
\left\|x_{n}-p\right\|=\left\|\left(1-\beta_{n}\right)\left[y_{n}-p+\gamma_{n}\left(u_{n}-y_{n}\right)\right]+\beta_{n}\left[T_{n}^{l_{n}} z_{n}-p+\gamma_{n}\left(u_{n}-y_{n}\right)\right]\right\| \longrightarrow d,
$$

as $n \rightarrow \infty$. Since $\lim _{n \rightarrow \infty} \gamma_{n}=0$ and $\left\{u_{n}\right\}$ is bounded, we have

$$
\begin{gathered}
\limsup _{n \rightarrow \infty}\left\|y_{n}-p+\gamma_{n}\left(u_{n}-y_{n}\right)\right\| \leq \limsup _{n \rightarrow \infty}\left[\left\|y_{n}-p\right\|+\gamma_{n}\left\|u_{n}-y_{n}\right\|\right] \leq d, \\
\limsup _{n \rightarrow \infty}\left\|T_{n}^{l_{n}} z_{n}-p+\gamma_{n}\left(u_{n}-y_{n}\right)\right\| \leq \limsup _{n \rightarrow \infty}\left[k_{l_{n}}\left\|z_{n}-p\right\|+\gamma_{n}\left\|u_{n}-y_{n}\right\|\right] \leq d .
\end{gathered}
$$

It follows from Lemma 1.7 that

$$
\lim _{n \rightarrow \infty}\left\|T_{n}^{l_{n}} z_{n}-y_{n}\right\|=0
$$

Thus,

$$
\begin{aligned}
\lim _{n \rightarrow \infty}\left\|z_{n}-y_{n}\right\| & =\lim _{n \rightarrow \infty}\left\|\bar{\alpha}_{n} y_{n}+\bar{\beta}_{n} T_{n}^{l_{n}} z_{n}+\bar{\gamma}_{n} \bar{u}_{n}-y_{n}\right\| \\
& =\lim _{n \rightarrow \infty}\left\|\bar{\beta}_{n}\left(T_{n}^{l_{n}} z_{n}-y_{n}\right)+\bar{\gamma}_{n}\left(\bar{u}_{n}-y_{n}\right)\right\|=0 .
\end{aligned}
$$

Similarly,

$$
\begin{aligned}
\lim _{n \rightarrow \infty}\left\|x_{n}-y_{n}\right\| & =\lim _{n \rightarrow \infty}\left\|\alpha_{n} y_{n}+\beta_{n} T_{n}^{l_{n}} z_{n}+\gamma_{n} u_{n}-y_{n}\right\| \\
& =\lim _{n \rightarrow \infty}\left\|\beta_{n}\left(T_{n}^{l_{n}} z_{n}-y_{n}\right)+\gamma_{n}\left(u_{n}-y_{n}\right)\right\|=0 .
\end{aligned}
$$

Moreover,

$$
\begin{aligned}
\left\|y_{n}-x_{n-1}\right\| & =\left\|\hat{\alpha}_{n} x_{n-1}+\hat{\beta}_{n} \hat{T}_{n}^{\hat{l}_{n}} y_{n}+\hat{\gamma}_{n} \hat{u}_{n}-x_{n-1}\right\| \\
& =\left\|\hat{\beta}_{n}\left(\hat{T}_{n}^{\hat{l}_{n}} y_{n}-x_{n-1}\right)+\hat{\gamma}_{n}\left(\hat{u}_{n}-x_{n-1}\right)\right\| \\
& \leq \hat{\beta}_{n}\left\|\hat{T}_{n}^{\hat{l}_{n}} y_{n}-x_{n-1}\right\|+\hat{\gamma}_{n}\left\|\hat{u}_{n}-x_{n-1}\right\| \longrightarrow 0, \text { as } n \longrightarrow \infty,
\end{aligned}
$$

since $\lim _{n \rightarrow \infty} \hat{\beta}_{n}=\lim _{n \rightarrow \infty} \hat{\gamma}_{n}=0$. As a result, we have

$$
\left\|x_{n}-x_{n-1}\right\| \leq\left\|x_{n}-y_{n}\right\|+\left\|y_{n}-x_{n-1}\right\| \longrightarrow 0, \quad \text { as } n \longrightarrow \infty .
$$

It forces

$$
\lim _{n \rightarrow \infty}\left\|x_{n}-x_{n+i}\right\|=0, \quad \text { for each } i=1,2, \ldots, N .
$$

On the other hand, we have

$$
\begin{aligned}
\left\|x_{n}-T_{n}^{l_{n}} x_{n}\right\| & \leq\left\|x_{n}-y_{n}\right\|+\left\|y_{n}-T_{n}^{l_{n}} z_{n}\right\|+\left\|T_{n}^{l_{n}} z_{n}-T_{n}^{l_{n}} x_{n}\right\| \\
& \leq\left\|x_{n}-y_{n}\right\|+\left\|y_{n}-T_{n}^{l_{n}} z_{n}\right\|+k_{l_{n}}\left\|z_{n}-x_{n}\right\| \longrightarrow 0, \quad \text { as } n \longrightarrow \infty
\end{aligned}
$$


As $n=l_{n} N+n(\bmod N)$ for $n>N$, we get

$$
n-N=\left(l_{n}-1\right) N+n(\bmod N),
$$

and hence $l_{n-N}=l_{n}-1$. Thus, we have

$$
T_{n}^{l_{n}-1}=T_{n-N}^{l_{n-N}}
$$

Consequently, we derive

$$
\begin{aligned}
\left\|x_{n}-T_{n} x_{n}\right\| \leq & \left\|x_{n}-T_{n}^{l_{n}} x_{n}\right\|+\left\|T_{n}^{l_{n}} x_{n}-T_{n} x_{n}\right\| \\
\leq & \left\|x_{n}-T_{n}^{l_{n}} x_{n}\right\|+K\left\|T_{n}^{l_{n}-1} x_{n}-x_{n}\right\| \\
= & \left\|x_{n}-T_{n}^{l_{n}} x_{n}\right\|+K\left\|T_{n-N}^{l_{n-N}} x_{n}-x_{n}\right\| \\
\leq & \quad\left\|x_{n}-T_{n}^{l_{n}} x_{n}\right\|+K\left[\left\|T_{n-N}^{l_{n-N}} x_{n}-T_{n-N}^{l_{n-N}} x_{n-N}\right\|\right. \\
& \left.\quad+\left\|T_{n-N}^{l_{n-N}} x_{n-N}-x_{n-N}\right\|+\left\|x_{n-N}-x_{n}\right\|\right] \\
\leq & \quad\left\|x_{n}-T_{n}^{l_{n}} x_{n}\right\|+K\left[(1+K)\left\|x_{n-N}-x_{n}\right\|\right. \\
& \left.\quad+\left\|T_{n-N}^{l_{n-N}} x_{n-N}-x_{n-N}\right\|\right] \longrightarrow 0, \quad \text { as } n \rightarrow \infty .
\end{aligned}
$$

This implies that for each $j=1,2, \ldots, N$,

$$
\begin{aligned}
\left\|x_{n}-T_{n+j} x_{n}\right\| & \leq\left\|x_{n}-x_{n+j}\right\|+\left\|x_{n+j}-T_{n+j} x_{n+j}\right\|+\left\|T_{n+j} x_{n+j}-T_{n+j} x_{n}\right\| \\
& \leq(1+K)\left\|x_{n}-x_{n+j}\right\|+\left\|x_{n+j}-T_{n+j} x_{n+j}\right\| \longrightarrow 0, \quad \text { as } n \longrightarrow \infty .
\end{aligned}
$$

Note that the closedness and convexity of $C$ imply the weak closedness of $C$. Let $\tilde{x} \in C$ be any weak cluster point of the bounded sequence $\left\{x_{n}\right\}$. Let $\left\{x_{n_{i}}\right\}$ be a subsequence of $\left\{x_{n}\right\}$ such that $x_{n_{i}} \rightarrow \tilde{x}$ weakly (see, e.g., [4, page 313]). Since the pool of mappings $\left\{T_{i}: 1 \leq i \leq N\right\}$ is finite, we may further assume (passing to a further subsequence if necessary) that for some integer $l \in\{1,2, \ldots, N\}, T_{n_{i}}=T_{l}$ for all $i \geq 1$. Then it follows from $(2.31)$ that for each $j=1,2, \ldots, N$,

$$
x_{n_{i}}-T_{l+j} x_{n_{i}} \longrightarrow 0, \quad \text { as } i \longrightarrow \infty,
$$

that is, for each $j=1,2, \ldots, N$,

$$
x_{n_{i}}-T_{j} x_{n_{i}} \longrightarrow 0, \quad \text { as } i \longrightarrow \infty .
$$

By Lemma 1.8, we can conclude that $\tilde{x} \in \bigcap_{j=1}^{N} F\left(T_{j}\right)$.

Theorem 2.4. In addition to the conditions in Theorem 2.3, assume further that $\varnothing \neq$ $\bigcap_{i=1}^{N} F\left(T_{i}\right) \subseteq \bigcap_{j=1}^{\hat{N}} F\left(\widehat{T}_{j}\right)$.

(a) If E satisfies Opial's condition, then $\left\{x_{n}\right\}$ converges weakly to an element of $\bigcap_{i=1}^{N} F\left(T_{i}\right)$.

(b) If one of $\left\{T_{i}\right\}_{i=1}^{N}$ is semicompact, then $\left\{x_{n}\right\}$ converges strongly to an element of $\bigcap_{i=1}^{N} F\left(T_{i}\right)$. 
Proof. We continue the argument in the proof of Theorem 2.3.

For (a), we claim that $\left\{x_{n}\right\}$ is weakly convergent. Were this false, there existed another subsequence $\left\{x_{n_{j}}\right\}$ of $\left\{x_{n}\right\}$ such that $x_{n_{j}} \rightarrow \bar{x} \in C$ weakly and $\bar{x} \neq \tilde{x}$. Utilizing the same argument as in Theorem 2.3, we can prove that $\bar{x} \in \bigcap_{j=1}^{N} F\left(T_{j}\right)$. Note that by Lemma 2.1, both $\lim _{n \rightarrow \infty}\left\|x_{n}-\tilde{x}\right\|$ and $\lim _{n \rightarrow \infty}\left\|x_{n}-\bar{x}\right\|$ exist. It follows from the Opial condition of $E$ that

$$
\begin{aligned}
\lim _{n \rightarrow \infty}\left\|x_{n}-\tilde{x}\right\| & =\liminf _{i \rightarrow \infty}\left\|x_{n_{i}}-\tilde{x}\right\| \\
& <\liminf _{i \rightarrow \infty}\left\|x_{n_{i}}-\bar{x}\right\|=\lim _{n \rightarrow \infty}\left\|x_{n}-\bar{x}\right\|=\liminf _{j \rightarrow \infty}\left\|x_{n_{j}}-\bar{x}\right\| \\
& <\liminf _{j \rightarrow \infty}\left\|x_{n_{j}}-\tilde{x}\right\|=\lim _{n \rightarrow \infty}\left\|x_{n}-\tilde{x}\right\| .
\end{aligned}
$$

This contradiction indicates that $\bar{x}=\tilde{x}$, and so $\left\{x_{n}\right\}$ converges weakly to $\tilde{x}$.

For (b), by (2.33), we can assume that a subsequence $\left\{x_{n_{i}}\right\}$ of $\left\{x_{n}\right\}$ exists such that $x_{n_{i}} \rightarrow \hat{x} \in \bigcap_{i=1}^{N} F\left(T_{i}\right)$ in norm. It then follows from Lemma 2.1 that

$$
\lim _{n \rightarrow \infty}\left\|x_{n}-\hat{x}\right\|=\lim _{i \rightarrow \infty}\left\|x_{n_{i}}-\hat{x}\right\|=0 .
$$

This completes the proof.

\section{Acknowledgments}

The first author was partially supported by the Teaching and Research Award Fund for Outstanding Young Teachers in Higher Education Institutions of MOE, China and the Dawn Program Foundation in Shanghai. The second and the third authors were partially supported by a grant from the National Science Council of Taiwan, NSC-94-2115-M110-004. NSC-95-2115-M-110-001.

\section{References}

[1] S. C. Bose, Weak convergence to the fixed point of an asymptotically nonexpansive map, Proceedings of the American Mathematical Society 68 (1978), no. 3, 305-308.

[2] S.-S. Chang, Y. J. Cho, and H. Zhou, Demi-closed principle and weak convergence problems for asymptotically nonexpansive mappings, Journal of the Korean Mathematical Society 38 (2001), no. $6,1245-1260$.

[3] K. Goebel and W. A. Kirk, A fixed point theorem for asymptotically nonexpansive mappings, Proceedings of the American Mathematical Society 35 (1972), no. 1, 171-174.

[4] G. Köthe, Topological Vector Spaces. I, Die Grundlehren der mathematischen Wissenschaften, vol. 159, Springer, New York, 1969.

[5] Z. Opial, Weak convergence of the sequence of successive approximations for nonexpansive mappings, Bulletin of the American Mathematical Society 73 (1967), 591-597.

[6] J. Schu, Weak and strong convergence to fixed points of asymptotically nonexpansive mappings, Bulletin of the Australian Mathematical Society 43 (1991), no. 1, 153-159.

[7] Z.-H. Sun, Strong convergence of an implicit iteration process for a finite family of asymptotically quasi-nonexpansive mappings, Journal of Mathematical Analysis and Applications 286 (2003), no. $1,351-358$.

[8] H.-K. Xu and R. G. Ori, An implicit iteration process for nonexpansive mappings, Numerical Functional Analysis and Optimization 22 (2001), no. 5-6, 767-773. 
[9] S. S. Zhang, On the iterative approximation problem of fixed points for asymptotically nonexpansive type mappings in Banach spaces, Applied Mathematics and Mechanics (English Edition) 22 (2001), no. 1, 25-34.

[10] Y. Zhou and S.-S. Chang, Convergence of implicit iteration process for a finite family of asymptotically nonexpansive mappings in Banach spaces, Numerical Functional Analysis and Optimization 23 (2002), no. 7-8, 911-921.

L. C. Ceng: Department of Mathematics, Shanghai Normal University, Shanghai 200234, China E-mail address: zenglc@shtu.edu.cn

N. C. Wong: Department of Applied Mathematics, National Sun Yat-sen University, Kaohsiung 804, Taiwan

E-mail address: wong@math.nsysu.edu.tw

J. C. Yao: Department of Applied Mathematics, National Sun Yat-sen University, Kaohsiung 804, Taiwan

E-mail address: yaojc@math.nsysu.edu.tw 